\title{
Morfologia do sistema genital feminino da paca (Cuniculus paca, Linnaeus, 1766)
}

\author{
Morphology of the female genital system of the paca (Cuniculus paca, Linnaeus, 1766)
}

\author{
Ana Carolina Gonçalves dos REIS ${ }^{1}$; Silvia Helena Brendolan GERBASI ${ }^{2}$; Carolina MARTINS ${ }^{3}$; Márcia \\ Rita Fernandes MACHADO $^{4}$; Cláudio Alvarenga de OLIVEIRA ${ }^{5}$ \\ ${ }^{1}$ Pós-graduanda do Programa de Pós-Graduação em Cirurgia da Faculdade de Ciências Agrárias e Veterinárias da Universidade \\ Estadual Paulista, Jaboticabal - SP, Brasil \\ ${ }^{2}$ Pós-graduanda do Centro Universitário Barão de Mauá, Ribeirão Preto - SP, Brasil \\ ${ }^{3}$ Graduanda do Curso de Medicina Veterinária da Faculdade de Ciências Agrárias e Veterinárias da Universidade Estadual Paulista, \\ Campus de Jaboticabal - SP, Brasil \\ ${ }^{4}$ Departamento de Morfologia e Fisiologia Animal da Faculdade de Ciências Agrárias e Veterinárias da Universidade Estadual \\ Paulista, Jaboticabal - SP, Brasil \\ ${ }^{5}$ Departamento de Reprodução Animal da Faculdade de Medicina Veterinária e Zootecnia da Universidade de São Paulo, São Paulo \\ - SP, Brasil
}

\begin{abstract}
Resumo
Foi caracterizada a morfologia macroscópica do genital feminino, de seis exemplares adultos de paca (Cuniculus paca), mediante dissecação das cavidades abdominal e pélvica imediatamente após o óbito. Os ovários apresentam forma ovoide, achatados dorso-ventralmente, de coloração amarela esbranquiçada com pequenos pontos avermelhados em sua superfície; têm localização sublombar, caudal aos rins; estão envoltos por uma rasa bolsa ovárica e fixados pelo mesovário; a tuba uterina é um órgão par, de aspecto sinuoso, contínua aos ovários, estando inserida na mesossalpinge e se estendendo até o início de cada corno uterino correspondente. Os cornos uterinos retilíneos fixam-se à parede abdominal pelo mesométrio e se unem pelo ligamento intercornual na altura da entrada da pelve, onde se posicionam dorsalmente à vesícula urinária; duas cevices estão presentes, embora o septo uterino que as separa seja incompleto, caracterizando presença de dois óstios uterinos internos e um único óstio uterino externo, considerando-se este útero como duplo incompleto. A vagina é um órgão tubular que se posiciona ventral ao reto e dorsal à vesícula urinária e à uretra, não se verificou a presença de vestíbulo e a vagina e a uretra não possuem ponto comum de convergência, abrindo-se, cada uma delas, diretamente na região vulvar, que se apresenta plana, com reduzidos lábios vulvares, apenas o clitóris de forma cônica é pouco proeminente e apresenta duas estruturas pontiagudas em sua região distal. Não se verificaram diferenças estatisticamente significativas nas mensurações realizadas nos ovários, tubas uterinas e cornos uterinos, ao se comparar os antímeros direito e esquerdo.
\end{abstract}

Palavras-chave: Reprodução. Roedor. Anatomia.

\begin{abstract}
The macroscopic morphology of genital tract of six adult female paca (Cuniculus paca) was studied through dissection of abdominal and pelvic cavities immediately after death. The ovaries have ovoid shape, dorsal-ventrally flattened, whitish yellow color with small red spots on its surface, located in sublombar situation, caudally to the kidneys, surrounded by a shallow bursa and fixed by the mesovarium; the oviduct is a pair of sinuous tubes, continuous with the ovaries, inserted in the mesosalpinx and extending up to the beginning of each correspondent uterine horn. The uterus horns are rectilinear and attached in the abdominal walls by the mesometrium, each horns extends caudally into the pelvic cavity and are linked by intercornual ligament, the ventral surface chiefly contacts the bladder; two cervices are present, although the uterine septum is incomplete, characterizing the presence of two internal uterine ostia and one external uterine ostium, considering this uterus as an incomplete duplex. The vagina is a tubular organ that is positioned ventrally to the rectum and dorsally to the urinary bladder and urethra; urethral duct is placed apart of the vaginal canal, characterizing the absence of the vestibulum; the vulva presents itself planed, with reduced vulvae lips, the clitoris is a conical structure with two prominent pointed structures at its distal region. There were no differences statistically significant in measurements made in the ovaries, oviducts and uterine horns, when comparing the right and left sides.
\end{abstract}

Keywords: Reproduction. Wild animal. Rodent. Anatomy.

Correspondência para:

Profa Dra. Márcia R. F. Machado.

Depto de Morfologia e Fisiologia Animal

Faculdade de Ciências Agrárias e Veterinárias, UNESP - Jaboticabal

Via de Acesso Prof Paulo Donato Castellane, s/n
CEP: 14884-900 - Jaboticabal - SP

e-mail: mrfmachd@fcav.unesp.br

Recebido: $17 / 12 / 2008$

Aprovado: 11/05/2011 


\section{Introdução}

A paca é o segundo maior roedor da fauna brasileira; apresenta carne de sabor apreciado tornando-se, portanto, vulnerável à exploração indiscriminada e, consequentemente, a correr risco de extinção ${ }^{1,2}$.

Além da importância comercial, o valor desses animais toma ainda, o âmbito da ciência, pois as pacas, que são roedores, podem ser uma boa alternativa para fins de pesquisa, colaborando com o desenvolvimento de investigações vitais ao homem e aos próprios animais, já que roedores e lagomorfos representam grupos de animais experimentais "ad hoc", em virtude de apresentarem aspectos característicos tais como tamanho adequado, preço acessível e curto período de prenhez ${ }^{3}$.

Poucas são as referências encontradas que mencionem aspectos anatômicos dos órgãos genitais femininos da paca, verificando-se apenas uma breve descrição de Matamoros ${ }^{2}$ sobre a anatomia e histologia do sistema reprodutor desses animais.

Sabendo-se que o conhecimento gerado a partir de estudos específicos à morfologia dos órgãos do sistema reprodutor de uma espécie é fundamental para o planejamento e estruturação dos mecanismos de sua preservação ao estabelecer padrões de referência de sua biologia reprodutiva, considera-se oportuna a realização de pesquisas relacionadas a este tema, na paca.

Dessa forma, a presente pesquisa fundamenta-se na necessidade de obter informações que possam subsidiar novos estudos, principalmente na área de reprodução animal, visando aproveitamento controlado e racional da espécie e consequentemente, sua preservação. Assim, objetivou-se descrever a anatomia macroscópica e a biometria do sistema genital feminino desse roedor.

\section{Material e Método}

Para a realização desta pesquisa foram utilizadas seis pacas (Cuniculus paca) fêmeas, todas adultas, com idade variando de 18 a 36 meses, provenientes do
Setor de Animais Silvestres do Departamento de Zootecnia da Faculdade de Ciências Agrárias e Veterinárias, Campus de Jaboticabal - UNESP, credenciado pelo IBAMA (cadastro número 482508), como criatório experimental. Todos os procedimentos foram realizados de acordo com o Consensus Recommendations on Effective Institutional Animal Care and Use Committees - NIH and USDA - publicado pelo Laboratory Animal Science, Special Issue, January 1987.

Os animais foram descartados, visando à seleção e manutenção do equilíbrio entre machos e fêmeas no criadouro, efetuando-se primeiramente, uma fase de tranquilização, à base de azaperone (Stresnil ${ }^{\circledR}$ - Janssen Pharmaceutica, São Paulo, SP/Brasil; $4 \mathrm{mg} / \mathrm{kg}$ ) por via intramuscular, seguida da aplicação intramuscular de sobredose de xilazina (Coopazine ${ }^{\oplus}$ - Schering-Plough Coopers Brasil Ltda., São Paulo, SP/Brasil) e cetamina (Vetaset ${ }^{\oplus}$ - Fort Dodge Saúde Animal Ltda., Campinas, SP/Brasil), cuja dosagem para a anestesia, nesses animais, é de $20 \mathrm{mg} / \mathrm{kg}$ e de $1,5 \mathrm{mg} / \mathrm{kg}$, respectivamente ${ }^{4}$.

Logo após o óbito, os animais foram posicionados em decúbito dorsal e, mediante incisão pré-retroumbilical inclusive com a abertura da sínfise pélvica, procedeu-se total abertura e exposição das cavidades abdominal e pélvica e suas estruturas. Em seguida, identificaram-se cada órgão em estudo, sua topografia e seus meios de sustentação, procedendo, então, ao registro fotográfico, "in situ", para a documentação.

Posteriormente, mediante dissecação individualizaram-se os órgãos em questão, observando-se, identificando-se e registrando-se fotograficamente as particularidades de cada um. Além disso, realizou-se a mensuração dos ovários (peso, comprimento, largura e diâmetro), das tubas uterinas (diâmetro e comprimento) e dos cornos uterinos (diâmetro e comprimento), com o auxílio de um paquímetro digital ("Starrett" cat. No. 727-6/150) e pesagem dos ovários em balança analítica (AL200C, Marte, Brasil). Os dados obtidos foram analisados pelo teste t pareado (GraphPad Prism 5), a fim de se verificar a ocorrência 
de diferenças estatisticamente significativas com nível de significância de $5 \%(p<0,05)$ entre as porções dos órgãos presentes nos antímeros direito e esquerdo.

A nomenclatura utilizada neste trabalho está de acordo com o lnternational Committee on Veterinary Gross Anatomical Nomenclature ${ }^{5}$.

\section{Resultados}

O sistema genital feminino da paca se constitui de ovários semelhantes em sua forma, dimensões e localização; tubas uterinas similares, contínuas aos ovários e que culminam nos retilíneos cornos uterinos análogos, os quais se continuam na cérvix quase dupla, pois o septo uterino que as separa é incompleto, caracterizando a presença de dois óstios uterinos internos e um único óstio uterino externo que se comunica com a vagina. A vagina chega diretamente na vulva, no "óstio vulvo-vaginal"; não se observa a presença de vestíbulo e a uretra termina no óstio uretral, também na região vulvar, bem próximo ao clitóris. A região vulvar não é proeminente, apenas o clitóris, de forma cônica, é levemente saliente e possui duas estruturas pontiagudas em sua região distal.

Quanto à biometria, verificou-se semelhança nos valores da média e desvio padrão referente às mensurações realizadas nos ovários, tubas uterinas e cornos uterinos, tanto ao se comparar os antímeros direito e esquerdo de um mesmo animal, quanto entre os animais (Tabelas 1, 2 e 3 ).

Os ovários da paca apresentam forma ovoide, achatados dorso-ventralmente, de coloração amarela esbranquiçada com pequenos pontos avermelhados em sua superfície, localizando-se em situação sublombar, caudalmente aos rins, na altura da terceira e quarta vértebras lombares. Cada ovário está envolto por uma rasa bolsa ovárica, que se caracteriza pela fusão extensiva do mesovário distal e do mesosalpinge e a fixação dessas gônadas é feita pelo mesovário, parte do ligamento largo do útero (Figura 1).

Tabela 1 - Média e erro padrão da média (EPM) dos dados morfométricos dos ovários direito (OD) e esquerdo (OE) da paca (Cuniculus paca): peso; comprimento (cranio-caudal); largura (látero-medial) e diâmetro - Jaboticabal - 2007

\begin{tabular}{ccccccccc}
\hline & \multicolumn{2}{c}{$\begin{array}{c}\text { Peso } \\
(\mathbf{g})\end{array}$} & \multicolumn{2}{c}{$\begin{array}{c}\text { Comprimento } \\
(\mathbf{c m})\end{array}$} & \multicolumn{2}{c}{$\begin{array}{c}\text { Largura } \\
(\mathbf{c m})\end{array}$} & \multicolumn{2}{c}{$\begin{array}{c}\text { Diâmetro } \\
(\mathbf{c m})\end{array}$} \\
& OD & OE & OD & OE & OD & OE & OD & OE \\
\hline Média & 1,70 & 1,68 & 2,03 & 2,02 & 1,39 & 1,38 & 3,78 & 3,78 \\
EPM & $\pm 0,005$ & $\pm 0,009$ & $\pm 0,032$ & $\pm 0,025$ & $\pm 0,005$ & $\pm 0,005$ & $\pm 0,008$ & $\pm 0,009$ \\
\hline
\end{tabular}

Tabela 2 - Média e erro padrão da média (EPM) dos dados biométricos do diâmetro (D) e comprimento $(\mathrm{C})$ das tubas uterinas direita (TD) e esquerda (TE) de paca (Cuniculus paca) - Jaboticabal - 2007

\begin{tabular}{ccccc}
\hline & \multicolumn{2}{c}{ Comprimento } & \multicolumn{2}{c}{$\begin{array}{c}\text { Diâmetro } \\
(\mathbf{m m})\end{array}$} \\
& TD & TE & TD & TE \\
\hline Média & 36,86 & 36,85 & 1,08 & 1,09 \\
EPM & $\pm 0,015$ & $\pm 0,021$ & $\pm 0,013$ & $\pm 0,02$ \\
\hline
\end{tabular}

Tabela 3 - Média e erro padrão da média (EPM) dos dados biométricos do comprimento (C) e diâmetro (D) dos cornos uterinos direito (CUD) e esquerdo (CUE) dos úteros de paca (Cuniculus paca) - Jaboticabal - 2007

\begin{tabular}{ccccc}
\hline & \multicolumn{2}{c}{ Comprimento } & \multicolumn{2}{c}{$\begin{array}{c}\text { Diâmetro } \\
(\mathbf{m m})\end{array}$} \\
& CUD & CUE & CUD & CUE \\
\hline Média & 96,32 & 96,32 & 25,87 & 26,15 \\
EPM & $\pm 0,032$ & $\pm 0,027$ & $\pm 0,228$ & $\pm 0,243$ \\
\hline
\end{tabular}


A média dos dados biométricos dos ovários direito e esquerdo foram, respectivamente, quanto ao peso de 1,70 g e 1,68 g; comprimento de $2,03 \mathrm{~cm}$ e $2,02 \mathrm{~cm}$; largura de $1,39 \mathrm{~cm}$ e $1,38 \mathrm{~cm}$ e diâmetro de $3,78 \mathrm{~cm}$ (Tabela 1).

Em todos os exemplares examinados observou-se que a tuba uterina é um órgão par, de aspecto sinuoso, localizada na região sublombar, contínua aos ovários, estendendo-se até ao início de cada corno uterino correspondente; inseridas em uma prega lateral em parte do mesovário denominada de mesossalpinge (Figura 1). Apresentam comprimento médio de $36,86 \mathrm{~mm}$ à direita e $36,85 \mathrm{~mm}$ à esquerda; diâmetro em média, de 1,08 mm à direita e 1,09 mm à esquerda (Tabela 2).

No que se refere ao útero da paca, verificou-se em todos os exemplares examinados, que este órgão localiza-se na região sublombar, caudal aos rins, contínuo aos ovários e às tubas uterinas, estendendo-se até a região próxima à entrada da pelve, onde se posiciona dorsalmente à vesícula urinária (Figura 1).

O útero desses roedores é formado por dois cornos uterinos retilíneos, cujo comprimento médio é de $96,32 \mathrm{~mm}$ e o diâmetro médio de $26,0 \mathrm{~mm}$ (Tabela 3). A parede medial de cada corno uterino converge caudalmente, formando um septo interno, o "velum"

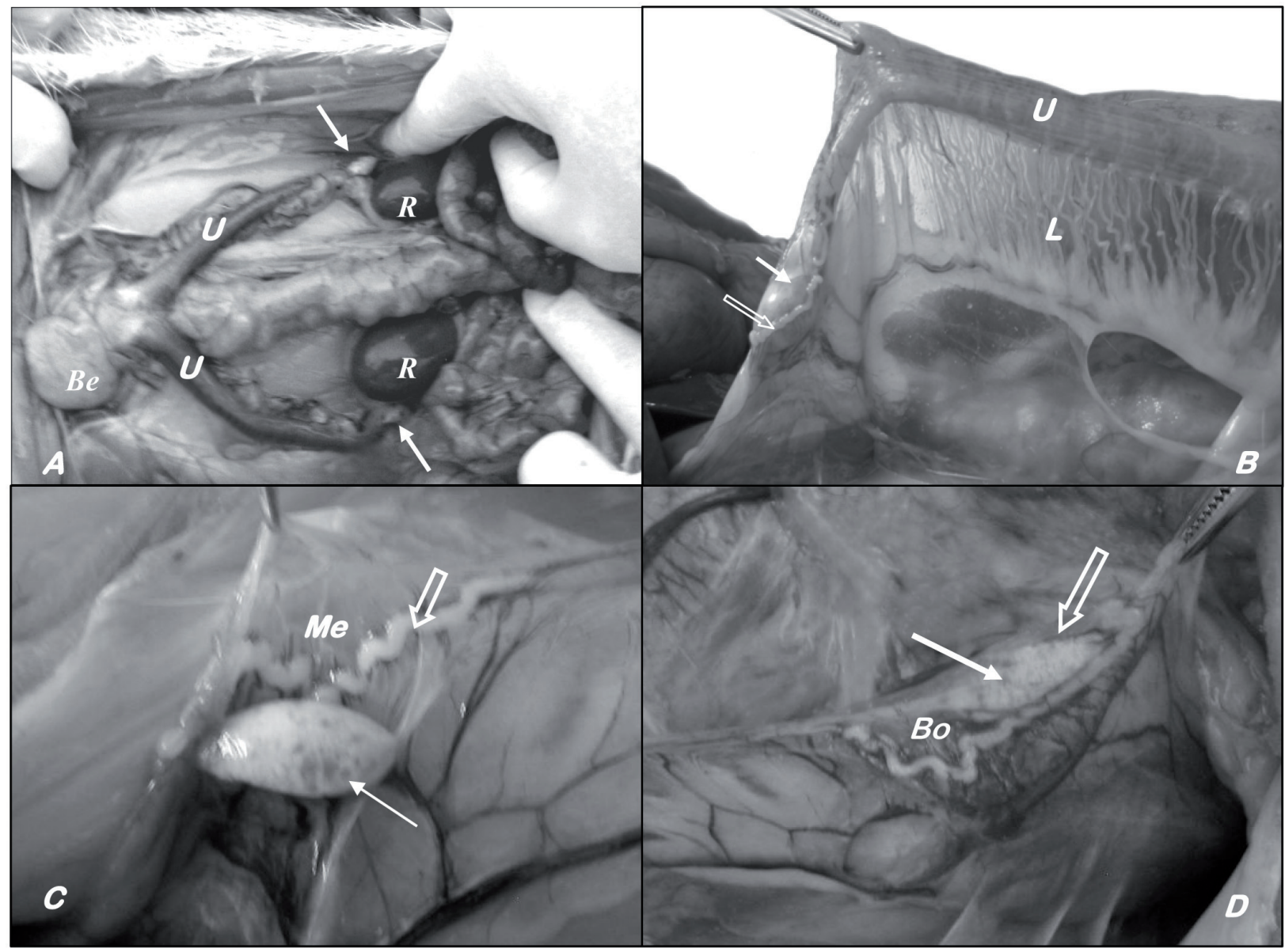

Figura 1 - Registros fotográficos do sistema genital feminino da paca (Cuniculus paca). Em $\boldsymbol{A}$ se observa a localização dos ovários com ovidutos (setas) e dos cornos úterinos (U), caudalmente aos rins (R), vesícula urinária (Be), na entrada da pelve. Em $\boldsymbol{B}$, se verifica o ovário e a rasa bolsa ovárica (seta); o oviduto (seta vazada), corno uterino (U) e ligamento largo do útero (L). Em $C$ nota-se a tuba uterina (seta vazada) no mesossalpinge (Me) e o ovário de formato ovoide, achatado dorso-ventralmente, com pequenos pontos em sua superfície (seta). Em $D$ se observa o ovário (seta), parcialmente na bolsa ovárica (Bo) e o infundíbulo do oviduto bem ligado à superfície ovariana (seta vazada) 
uterino, que embora incompleto, determina a formação de dois canais cervicais quase individuais com a presença de dois óstios uterinos internos e apenas um óstio uterino externo que se abre na vagina (Figura 2).

Os cornos uterinos se interligam pelo ligamento intercornual e fixam-se à parede abdominal por meio do mesométrio, uma das extensões do ligamento largo do útero (Figura 1).

A vagina da paca se inicia em continuidade ao útero, logo após o colo uterino, estendendo-se até a vul- va, onde se abre externamente. Neste roedor, a vagina localiza-se inicialmente na cavidade abdominal prosseguindo em direção à cavidade pélvica, a qual adentra, posicionando-se ventralmente ao reto e dorsal à vesícula urinária e uretra. $\mathrm{Na}$ extremidade proximal da vagina, circundando a parte vaginal do colo uterino, verifica-se uma projeção em fundo de saco cego, o fórnix vaginal (Figura 2). Foram observadas pregas longitudinais em toda a parede vaginal interna. Nesses roedores não se verificou convergência da uretra

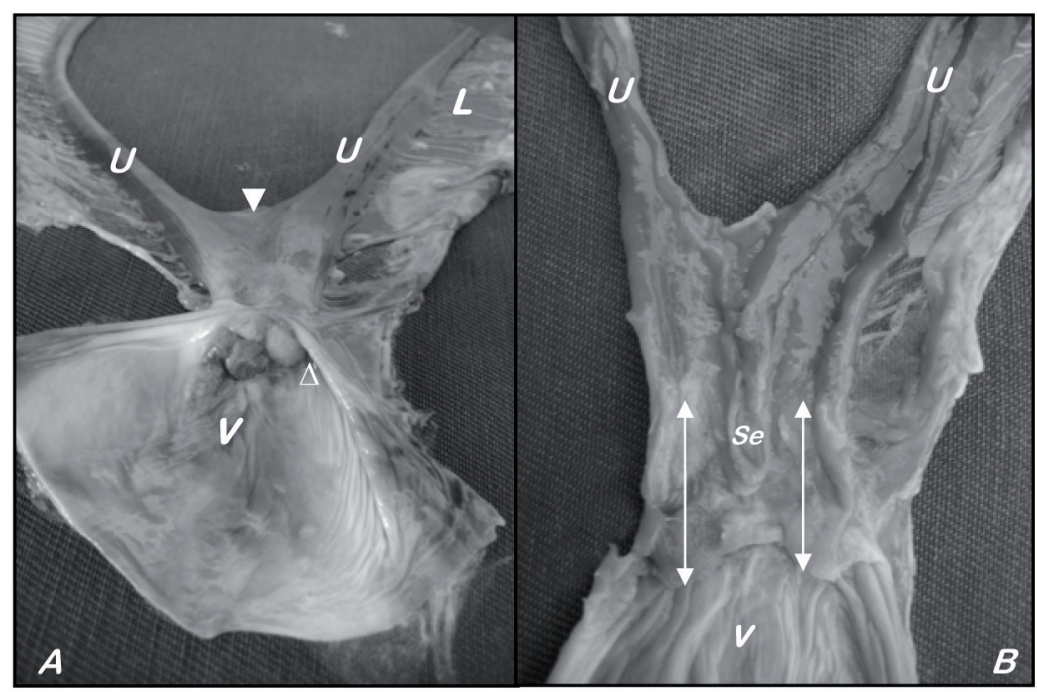

Figura 2 - Em $\boldsymbol{A}$, registro fotográfico de peça de útero removida de Cuniculus paca, indicando parte dos cornos uterinos direito e esquerdo $(U)$; ligamento intercornual $(\boldsymbol{\nabla})$; parte do mesométrio $(L)$; vagina $(V)$, fórnix $(\Delta)$. Em $\boldsymbol{B}$ verifica-se parte dos cornos uterinos $(U)$ seccionados; o septo interno incompleto $(\mathrm{Se})$ entre as cérvices (setas), parte da vagina $(\mathrm{V})$ ressaltando as pregas longitudinais da mucosa vaginal

com a vagina; a uretra se abre independentemente, junto ao clitóris, caracterizando a ausência de vestíbulo (Figura 3).

A vulva apresenta-se como uma região relativamente plana; delimita-se dorsalmente por uma depressão logo abaixo do ânus e, ventralmente pela projeção do clitóris. O óstio "vulvo-vaginal”, quando aparente, se mostra como um orifício transversal de reduzidas dimensões, contornado por pequenos lábios vulvares (Figura 3).
O clitóris apresenta-se, externamente, como uma estrutura proeminente de forma cônica e revestida por pele; em seu ápice pode ser observado pequeno orifício, o óstio uretral, ladeado por um tufo de pelos longos. Internamente encontra-se o corpo do clitóris, um cordão de consistência firme, posicionado paralela e ventralmente em relação à uretra. Ressalta-se que na extremidade distal deste órgão, há a presença de duas estruturas pontiagudas semelhantes a espinhos, 
só detectáveis quando a pele que recobre o clitóris é tracionada cranialmente, expondo-as (Figura 3).

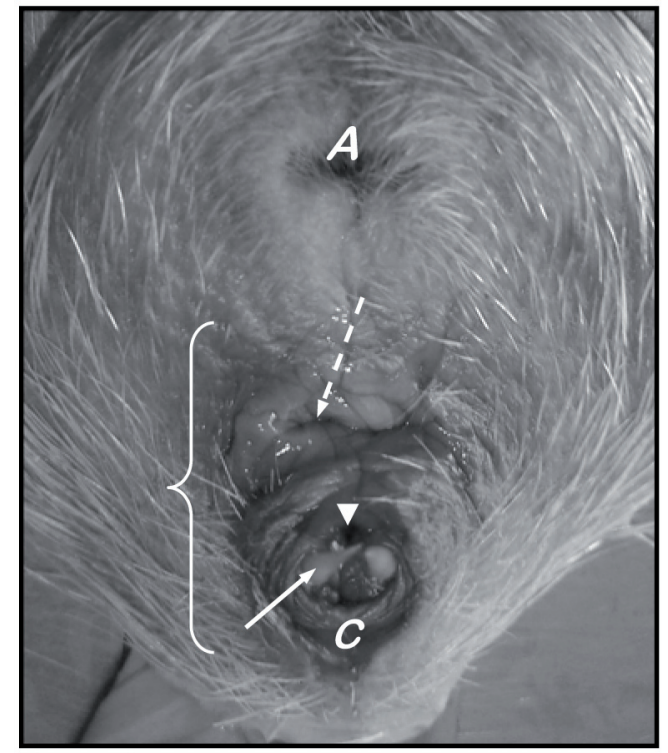

Figura 3 - Imagem fotográfica em vista dorsal de peça individualizada onde se observa o ânus $(A)$, o clitóris $(C)$ e a região vulvar (\{). O prepúcio do clitóris está parcialmente removido, expondo duas estruturas semelhantes a espinhos (seta) e o óstio uretral (cabeça de seta), verifica-se também o óstio vaginal (seta pontilhada)

\section{Discussão}

Quanto às observações referentes à localização dos ovários da paca, ora verificados, estas coincidem aos achados de Dumas ${ }^{6}$ para a cobaia, Dumas ${ }^{6}$ e Cook $^{7}$ para camundongos, Greene ${ }^{8}$ e Chiasson ${ }^{9}$ para o rato albino, Weir ${ }^{10}$, Guimarães, Matos e Vale ${ }^{11}$ e Almeida et al. ${ }^{12}$ para a cutia (Dasyprocta aguti, Dasyprocta prymnolopha e Dasyprocta aguti, respectivamente) e Matamoros $^{2}$ para a paca. A observação sobre a esqueletopia deste órgão, à direita e à esquerda da coluna vertebral (entre a terceira e quarta vértebras lombares), assemelha-se, em parte, às descrições de Almeida et al. ${ }^{12}$, que relatam para a cutia (Dasyprocta aguti), posição dos ovários entre a quarta e quinta vértebras lombares. $\mathrm{O}$ formato ovalado, achatado dorso-ventralmente, ob- servado no ovário da paca, corroboram as descrições de Dumas ${ }^{6}$ para a cobaia, de Weir ${ }^{10}$ e Almeida et al. ${ }^{12}$ para a cutia, de Weir e Rowlands ${ }^{13}$ para a maioria dos roedores histricomorfos e de Matamoros ${ }^{2}$, para a paca (Cuniculus paca). Esta característica difere do camundongo, que apresenta ovário arredondado ${ }^{6}$; do rato, cujo ovário aparece como uma massa de folículos ${ }^{8,9}$, da capivara, que tem ovário com formato irregular ${ }^{14} \mathrm{e}$ no roedor viscacha da planície, cuja superfície ovariana é aumentada pela presença de sulcos ${ }^{13}$.

A coloração amarela esbranquiçada, verificada neste estudo, assemelha-se às descrições relativas à paca ${ }^{2}$ e à cutia ${ }^{12}$, quanto os pequenos pontos avermelhados em sua superfície, provavelmente referem-se às pequenas áreas translúcidas, sugestivas da presença de folículos em diferentes ordens, conforme achados semelhantes às verificações de Weir e Rowlands ${ }^{13}$, para a maioria dos roedores histricomorfos, de Matamoros ${ }^{2}$ para a paca e de Almeida et al. ${ }^{12}$ para a cutia.

Não se verificou, nesta pesquisa, o ovário imerso em tecido adiposo, como no camundongo ${ }^{6,7}$, rato albino $^{8}$ e cutia ${ }^{11,12}$. Os elementos suspensores dos ovários, verificados para a paca, coincidem com as descrições de Weir e Rowlands ${ }^{13}$, para os histricomorfos de um modo geral, as de Ojasti ${ }^{14}$, para a capivara e as de Almeida et al. ${ }^{12}$ para a cutia. Assim como está descrito para a cutia, Almeida et al. ${ }^{12}$ não consideram uma bolsa ovárica o envolvimento do ovário pelo mesossalpinge.

Relativamente aos dados biométricos do ovário, ora observados, esses dados aproximam-se aos encontrados para a paca $^{2}$, mas são inferiores aos expostos para a cutia por Weir ${ }^{10}$ e por Silva e Perdomo ${ }^{15}$ para a capivara e superiores aos apresentados para a cutia por Almeida et al. ${ }^{12}$.

A topografia e a forma da tuba uterina da paca, ora observada, assemelham-se às descrições referentes a estes aspectos na cobaia, que apresenta tuba também sinuosa e que se insere na bursa ovárica ${ }^{16}$, na paca, constituida por um tubo fino e contorcido que man- 
tem contato com a superfície medial do ovário e do mesovário ${ }^{2}$ e nos esquilos, que apresenta tuba ovárica com corpo sinuoso localizando-se lateral e medialmente aos ovários ${ }^{17}$, também o comprimento médio da tuba da paca, assemelha-se mais ao descrito para os esquilos, de aproximadamente $40 \mathrm{~mm}^{17}$.

Embora não se tenha dissecado e observado, com auxílio de lupa, o infundíbulo do oviduto da paca parecia estar totalmente ligado à superfície ovariana conforme ocorre no porco espinho ${ }^{18}$.

Ao se analisar isoladamente o útero da paca, constatou-se a presença de canal cervical duplo, com dois óstios uterinos internos e apenas um externo, diferindo das premissas de que o útero é duplo quando há a presença de duas cérvices ou presença de duas cérvices que entram na vagina independentemente ${ }^{19,20,21,22}$. Dessa forma não se considerou o útero da paca $(\mathrm{Cu}$ niculus paca) como duplo, diferindo dos relatos para a maioria dos roedores ${ }^{14,19,20,21,22,23,24,25,26,27,28,29}$ e sim útero duplo incompleto.

Também não se classifica o útero da paca como bicorne onde não ocorre septação, caracterizando a presença de dois cornos, um corpo e uma cérvix, como descrito por Nickel, Schummer e Seiferle ${ }^{19}$; Liem et al. ${ }^{20}$; Dyce, Sack e Wensing ${ }^{21}$ e Hildebrand; Goslow ${ }^{22}$. Esta classificação foi conferida ao hamster ${ }^{24}$, cobaias ${ }^{16,25}, \mathrm{Cu}$ niculus paca $^{30}$, camundongo ${ }^{24}$, e porco-espinho ${ }^{18}$.

Tal qual os relatos de Moura et al. ${ }^{31}$, para a cutia, pode-se considerar o útero da paca como duplo incompleto, já que nesses animais não se verifica dois óstios uterinos externos abrindo-se na vagina.

A vagina da paca, assim como no roedor Myocastor coypus ${ }^{28}$, é um órgão tubular que se posiciona ventral ao reto e dorsal à vesícula urinária e à uretra, iniciando-se em continuidade ao útero, logo após o colo uterino; seu segmento cranial, envolto por peritônio, localiza-se na cavidade abdominal e seu segmento caudal, recoberto pela túnica adventícia, encontra-se na cavidade pélvica. Em sua porção cranial observa-se uma projeção em fundo de saco cego, o fórnix vaginal, estrutura também encontrada no Athererus africanus ${ }^{18}$.

A mucosa vaginal da paca apresenta pregas longitudinais em toda a sua extensão, de forma similar ao coipu, ao coelho e ao porco-espinho do Gabão ${ }^{18,28,32}$. A presença de pregas na mucosa da vaginal ocorre quando a vagina, considerada órgão copulatório e canal do parto, está inativa ${ }^{21}$.

Na paca, a vagina e a uretra não possuem ponto comum de convergência, abrindo-se, cada uma delas, diretamente na vulva, caracterizando assim ausência de vestíbulo. Tal atributo foi também descrito para Cuniculus paca ${ }^{2}$, Hydrochoerus hidrochaeris ${ }^{33,34}$, Myocastor coypus ${ }^{28}$, Athererus africanus ${ }^{18}$ e Scapanus latimanus $^{35}$. Nos roedores ocorre um desenvolvimento alternativo do seio urogenital primitivo, assim a uretra das fêmeas desemboca fora da vagina e estão presentes nesta espécie três aberturas separadas: a uretra, a vagina e o orifício anal ${ }^{36}$.

A região vulvar da paca, tal qual na cobaia ${ }^{34}$, se encontra delimitada dorsalmente por uma depressão logo abaixo do ânus, ventralmente pelo clitóris e lateralmente por reduzidos lábios vulvares; apresenta-se como uma região relativamente plana, sem projeções, de forma diferente à da vulva dos coelhos ${ }^{26,37}$ e similar ao relatado para Hydrochoerus hidrochaeris ${ }^{34}$.

O clitóris, externo à vagina, apresenta-se cônico e proeminente e juntamente a ele ocorre a desembocadura da uretra; estrutura semelhante foi descrita por Matamoros ${ }^{2}$, por Felipe et al. ${ }^{28}$ e por Rubenstein et al. ${ }^{35}$, para Cuniculus paca, Myocastor coypus e toupeira da espécie Scapanus latimanus, respectivamente, diferindo ainda da cobaia, na qual o clitóris se apresenta grande, macio, carnoso e linguiforme ${ }^{34}$.

Quanto à presença de duas estruturas pontiagudas observadas na região distal do clitóris, este é um achado para o qual, na literatura consultada, não foi encontrada alusão análoga em fêmeas de outros roedores, assim como citações ou hipóteses sobre sua morfofuncionalidade. Porém, Pocock ${ }^{34}$ e 
Mondolfi ${ }^{1}$, ao descreverem o macho de Dasyprocta e Cuniculus paca, respectivamente, relataram estruturas semelhantes quando se referiram à porção distal do pênis, embora não façam alusão quanto à sua função. Baseando-se nas conjecturas de Arnold $^{38}$ e Wake ${ }^{39}$, ao inferirem que as estruturas diferenciadas, encontradas nos órgãos copulatórios de lacertídeos e anfíbios, podem estar relacionadas ao reconhecimento do companheiro, a um mecanismo de chave fechadura, ou até mesmo a um mecanismo físico de isolamento da espécie gerado por conflito mecânico. Estas evidências, porém, não lançam luz sobre a questão da morfofuncionalidade do peculiar clitóris da paca, para que uma hipótese consistente seja formulada. Deve-se considerar que muitas vezes as estratégias reprodutivas dos vertebrados relacio-

\section{Referências}

1. MONDOLFI, E. La laca o paca. Defensa de la Naturaleza. Caracas, v. 2, n. 5, p. 4-16, 1972.

2. MATAMOROS, Y. Investigaciones preliminares sobre la reproduccion, comportamiento, alimentacion y manejo tapezcuinte (Cuniculus paca, Brisson) en cautiverio. In: CONGRESSO LATINOAMERICANO DE ZOOLOGIA, 1982, Salinas. Anais... Salinas: Zoologia Neotropical, 1982. p. 961-992.

3.BJÖRKMAN, N.; DANTZER, V.; LEISER, R. Comparative placentation in laboratory animals a review. Scandinavian Journal of Animal Science, v. 16, p. 129-158, 1989.

4. OLIVEIRA, F. S.; TONIOLLO, G. H.; MACHADO, M. R. F.; PAURA, D. Hemi-ovariossalpingohisterectomia em pacas prenhes e posterior ocorrência de prenhez (Agouti paca, linnaeus, 1766). Ciência Rural, v. 33, n. 3, p. 547-551, 2003.

5.INTERNATIONAL COMMITTEE ON VETERINARY GROSS ANATOMICAL NOMENCLATURE. Nomina Anatomica Veterinaria. 5. ed. Hannover: International Committee on Veterinary Gross Anatomical Nomenclature, 2005. 166 p. Disponível em: <http://www.wava-amav.org/ dowloads/nav_2005.pdf $>$. Acesso em: 20 jul. 2010.

6. DUMAS, J. Les animaux de laboratoire. Paris: Médicales Flammarion, 1953.711 p.

7. COOK, M. J. The anatomy of the laboratory mouse. London: Academic Press, 1965. 143 p.

8. GREENE, E. C. Anatomy of the rat. Philadelphia: American Philosophical Society, 1955. 370 p.

9. CHIASSON, R. B. Laboratory anatomy of the white rat. 2. ed. Arizona: Wm. C. Brown, 1969. 81 p.

10.WEIR, B. Some observations on reproduction in the female agouti, Dasyprocta aguti. Journal of Reproduction and Fertility, v. 24, n. 2, p. 203-211, 1971. nam-se direta ou indiretamente com a estrutura do sistema genital ${ }^{22}$.

\section{Conclusões}

O sistema genital feminino da paca se constitui de ovários, tubas uterinas, útero duplo incompleto, vagina e região vulvar, onde se projeta o clitóris e este apresenta duas estruturas cônicas pontiagudas.

Não se observou a presença de vestíbulo da vagina, a uretra desemboca fora da vagina, verificando-se que a uretra, a vagina e o orifício anal abrem-se separadamente para o exterior.

Não se constatou diferenças estatisticamente significativas nos valores referentes às mensurações realizadas nos ovários, tubas uterinas e cornos uterinos, ao se comparar os antímeros direito e esquerdo.

11.GUIMARÃES, D. A.; MATOS, E.; VALE, W. G. Estudo morfológico do sistema genital feminino de cutia (Dasyprocta prymnolopha, Rodentia: Cavidae). Revista Brasileira de Ciências Morfológicas, v. 11, n. 2, p. 167-171, 1994.

12.ALMEIDA, M. M. de; CARVALHO, M. A. M. de; CAVALCANTE FILHO, F.; MIGLINO, M. A.; MENEZES, D. J. A. de. Estudo morfológico e morfométrico do ovário de cutias (Dasyprocta aguti, Linaeus, 1766). Brazilian Journal of Veterinary Research and Animal Science, v. 40, n. 1, p. 55-62, 2003. Disponível em: <http://www.scielo.br/scielo. php? script $=$ sci_arttext\&pid $=$ S1413-95962003000100006\&lng $=$ en $\& \mathrm{nrm}=\mathrm{iso}>$. Acesso em: $12 \mathrm{dec} .2008$.

13.WEIR, B. J.; ROWLANDS, I. W. Functional anatomy of the hystricomorph ovary. In: SYMPOSIUM OF THE ZOOLOGICAL SOCIETY OF LONDON, 34, 1974. [s.l.], Anais... [1974]. p. 303-332.

14. OJASTI, J. Estudio biológico del chiguiiro o capibara. Caracas: FONAIAP, 1973. 277 p.

15.SILVA, C. L.; PERDOMO, F. Algunos aspectos anatómicos e histológicos del sistema genital femenino del chiguire (Hidrochoerus hidrochaeris). Revista da Faculdade de Ciências Veterinárias da Universidade Central da Venezuela, v. 30, n. 1/8, p. 89-97, 1983.

16. COOPER, G.; SCHILLER, A. L. Anatomy of the guinea pig. Cambridge: Harvard University Press, 1975. 328 p.

17.NIKOLIC, Z.; BLAGOJEVIK, Z.; KATAKANIC, D.; ROGOZARSKI, D. The ovary and the oviduct of the ground squirrel (Citellus citellus). Acta Veterinaria, v. 43, n. 2-3, p. 171-177, 1993.

18.MAYOR，P.; LÓPEZ-BÉJAR， M.; JORI，F.; RUTLLANT, J.; LÓPEZ-PLANA, C.; LÓPEZ-GATIUS, F. Anatomicohistological characteristics of the genital tubular organs of the female Brush-tailed Porcupine (Athererus 
africanus, Gray 1842) from Gabon. Anatomia Histologia Embryologia, v. 31, p. 355-361, 2002.

19.NICKEL, R.; SCHUMMER, A.; SEIFERLE, E. The viscera of the domestic mammals. $2^{\text {nd }}$ ed. Berlim: Verlag Paul Parey, 1979. p. 351-389.

20.LIEM, K.; BEMIS, W.; WALKER, W. F.; GRANDE, L. Functional anatomy of the vertebrates: an evolutionary perspective. $3^{\text {rd }}$ ed. Belmont: Thomson Learning, 2001. 703 p.

21.DYCE, K. M.; SACK, W. O.; WENSING, C. J. G. Tratado de anatomia veterinária. 3. ed. Rio de Janeiro: Guanabara Koogan, 2004. p. 164-207.

22. HILDEBRAND, M.; GOSLOW, J. R. Análise da estrutura dos vertebrados. 2. ed. São Paulo: Atheneu, 2006. 637 p.

23. ATWOOD, W. J. Comparative anatomy. 2. ed. St. Louis: The C. V. Mosby Company, 1955. 424 p.

24. SMALLWOOD, J. E. A guided tour of veterinary anatomy. Philadelphia: W.B Saunders Company, 1992. p. 340-365.

25.BESSELSEN, D. G. Biology of laboratory rodents. 2002. Disponível em: <http://www.ahc.arizona.edu/uac/notes/ classes/rodentbio/rodentbio.html>. Acesso em: 15 fev. 2005.

26. VAN PRAAG, E. Reproductive tract of the female rabbit. 2003. Disponível em: <http://www.medirabbit.com/EN/Uro gen_diseases/generalities/female_organ.PDF $>$. Acesso em: $2 \overline{8}$ jun. 2007.

27. GUIMARÃES, D. A. Algumas características reprodutivas da cutia fêmea (Dasyprocta prymnolopha) criada em cativeiro. 1993. 89 f. Dissertação (Mestrado em Biologia) - Universidade Federal do Pará, Museu Paraense Emílio Goeldi, Belém, 1993.

28. FELIPE, A. E.; CASTRO, A.; CALLEJAS, S.; CABODEVILA, J. A. Morphological study of the vagina of the Myocastor coypus (coypu). Revista Chilena de Anatomía, v. 18, p. 231-235, 2000.

29. COSTA, D. S.; PAULA, T. A. R.; FONSECA, C. C.; NEVES, M. T. D. Reprodução de capivaras. Arquivos de Ciências Veterinárias e Zoologia, v. 5, n. 1, p. 111-118, 2002.
30.MATAMOROS, Y. Anatomía e histología del sistema reproductor del tepezcuinte (Cuniculus paca). Revista de Biología Tropical, v. 29, n. 1, p. 155-164, 1981.

31.MOURA, S. G.; CARVALHO, M. A. M.; ARAÚJO, W. R.; VIEIRA, R. J.; ALMEIDA, M. M.; OLIVEIRA, M. F. Proposta de classificação para útero da cutia (Dasyprocta aguti, Linnaes, 1766). Revista Brasileira de Reprodução Animal, v. 27, n. 2, p. 284-285, 2003.

32.KANAGAWA, H.; HAFEZ, W. C.; PITHFORD, W. C.; BAECHLER, C. A.; BARNHART, M. I. Surface patterns in the reproductive tracts of the rabbit observed by scanning electron microscopy. The Anatomical Record, v. 197, p. 205-226, 1972.

33.HUNTER, J.; OWEN, R. Essay observations on natural history, anatomy, physiology, psychology and geology. London: John van Voorst Paternoste Row, 1861. 403 p.

34.POCOCK, R. I. On the external characters of some hystricomorph rodents. Proceeding Zoological Society, v. 25, p. $365-427,1992$.

35. RUBENSTEIN, N. M.; CUNHA, G. R.; WANG, Y. Z.; KAMPBELL, K. L.; CONLEY, A. J.; CATANIA, K. C.; GLICKMAN, S. E.; PLACE, N. J. Variation in ovarian morphology in four species of New World moles with a peniform clitoris. Reproduction, v. 126, p. 713-719, 2003.

36.POPESKO, P.; RAJTOVÀ, V.; HORAK, J. A color atlas of anatomy of small laboratory animals. London: Wolfe Publishing Ltd, 1992. $2 \mathrm{v}$.

37.BARONE, R. Anatomie comparee des mammifères domestiques. Paris: Vigot, 1990. v. 4, chap. 3, 441 p.

38. ARNOLD, N. E. The hemipenis of lacertid lizards (Reptilia: Lacertidae): structure, variation and systematic implications. Journal of Natural History, v. 20, n. 5, p. 1221-1257, 1986.

39. WAKE, M. H. Cartilage in the Cloaca: Phallodeal Spicules in Caecilians (Amphibia: Gymnophiona). Journal of Morphology, v. 237, p.177-186, 1998. 